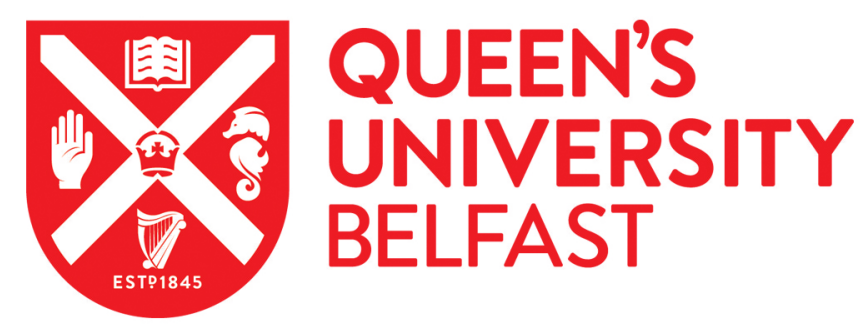

\title{
Dissipative equation of motion for electromagnetic radiation in quantum dynamics
}

Bustamante, C., Gadea, E., Horsfield, A. P., Todorov, T., Gonzalez Lebrero, M., \& Scherlis, D. A. (2021).

Dissipative equation of motion for electromagnetic radiation in quantum dynamics. Physical Review Letters. https://doi.org/10.1103/PhysRevLett.126.087401

Published in:

Physical Review Letters

Document Version:

Peer reviewed version

Queen's University Belfast - Research Portal:

Link to publication record in Queen's University Belfast Research Portal

Publisher rights

Copyright 2021 American Physical Society. This work is made available online in accordance with the publisher's policies. Please refer to any applicable terms of use of the publisher.

\section{General rights}

Copyright for the publications made accessible via the Queen's University Belfast Research Portal is retained by the author(s) and / or other copyright owners and it is a condition of accessing these publications that users recognise and abide by the legal requirements associated with these rights.

Take down policy

The Research Portal is Queen's institutional repository that provides access to Queen's research output. Every effort has been made to ensure that content in the Research Portal does not infringe any person's rights, or applicable UK laws. If you discover content in the Research Portal that you believe breaches copyright or violates any law, please contact openaccess@qub.ac.uk. 


\title{
Dissipative equation of motion for electromagnetic radiation in quantum dynamics
}

\author{
Carlos M. Bustamante, ${ }^{1}$ Esteban D. Gadea, ${ }^{1}$ Andrew Horsfield* ${ }^{2}$ Tchavdar \\ N. Todorov*, ${ }^{3}$ Mariano C. González Lebrero, ${ }^{1}$ and Damián A. Scherlis*1 \\ ${ }^{1}$ Departamento de Química Inorgánica, Analítica y Química Física/INQUIMAE, \\ Facultad de Ciencias Exactas y Naturales, Universidad de Buenos Aires, Buenos Aires (C1428EHA) Argentina \\ ${ }^{2}$ Department of Materials, Thomas Young Centre, Imperial College London, \\ South Kensington Campus, London, SW7 2AZ, United Kingdom \\ ${ }^{3}$ Atomistic Simulation Centre, School of Mathematics and Physics, \\ Queen's University Belfast, Belfast BTr 1NN, United Kingdom
}

(Dated: January 24, 2021)

\begin{abstract}
The dynamical description of the radiative decay of an electronically excited state in realistic many-particle systems is an unresolved challenge. In the present investigation electromagnetic radiation of the charge density is approximated as the power dissipated by a classical dipole, to cast the emission in closed form as a unitary single-electron theory. This results in a formalism of unprecedented efficiency, critical for ab-initio modelling, which exhibits at the same time remarkable properties: it quantitatively predicts decay rates, natural broadening, and absorption intensities. Exquisitely accurate excitation lifetimes are obtained from time-dependent DFT simulations for $\mathrm{C}^{2+}, \mathrm{B}^{+}$and $\mathrm{Be}$, of $0.565,0.831$ and $1.97 \mathrm{~ns}$ respectively, in accord with experimental values of $0.57 \pm 0.02,0.86 \pm 0.07$ and $1.77-2.5$ ns. Hence, the present development expands the frontiers of quantum dynamics, bringing within reach first-principles simulations of a wealth of photophysical phenomena, from fluorescence to time-resolved spectroscopies.
\end{abstract}

Introduction. - Time-dependent electronic structure simulations are currently applied to fundamental phenomena at the nanoscale, from spectroscopy [1-4] and photochemistry [5-8] to quantum conductance [9-13] and energy transfer in molecules and materials $[2,14-17]$. Despite the insight that these simulations provide, they lack an important ingredient: the radiative energy dissipation for any charge density evolving in time. This missing piece will affect the electron dynamics in accordance with the magnitude and rate of the density fluctuations. Without it, the energy absorbed during the excitation of a molecule at zero temperature will remain in the system in the form of undamped dipole oscillations, indefinitely in time [18], which is unphysical.

Different schemes have been proposed to account for the radiative decay or spontaneous emission in electron dynamics. Treatments rooted in quantum electrodynamics have been devised to propagate jointly the electronic density and the quantized electromagnetic field [19-23]. Such approaches are important in situations with strong photon-electron coupling as occurs under intense laser fields, or the interaction of light with molecules or quantum dots in optical cavities [24, 25]. However, the high dimensionality of the Hilbert space containing the photon modes demands a truncation or simplification of the electromagnetic quantum field for numerical tractability. A recent development is the application of the exact factorization approach to light-matter interaction [26-28].

An alternative route to model light-matter coupling is to describe the electrons quantum-mechanically, and the radiation field in terms of classical Maxwell equations. The Maxwell-Bloch equations, and their generalization the Maxwell-Liouville equations [29-33], or the Ehrenfest method [34], illustrate this semiclassical treatment. In this framework the classical electric and magnetic fields evolve self-consistently with the electronic density matrix. Most implementations of these methods have been limited to just a few - often just two - level systems. An overview and comparative analysis of these approaches have been recently contributed by Nitzan, Subotnik, and co-authors [34-36]. In particular, these researchers have proposed a correction to Ehrenfest dynamics that, by construction, reproduces spontaneous emission according to the radiative decay rate deduced from Fermi's golden rule (FGR) [37].

Strategies that do not fit exactly in these groups have been explored very recently [38, 39]. Furthermore, we draw attention to Rashkovskiy's work on the non-linear Schrödinger equation, where thermal radiation and spontaneous emission are described without energy quantization in a classical field framework [40, 41].

In the present study, starting from a mixed quantumclassical Lagrangian and a dipolar approximation, we formulate an equation of motion that describes radiative dissipation in electronic systems without empirical or fitted parameters. Despite its simplicity, this formalism quantitatively captures much of the physics of radiation emission and absorption, including the rates of exponential decay, the natural broadening, and the relative intensities of absorption bands. Transparent and inexpensive, this development introduces an alternative approach of unprecedented accuracy and tractability that overcomes the limitations of current methods, realizing a goal that appeared remote: inclusion of the lightmatter interaction in first-principles time-dependent simulations of many-electron systems. Here we illustrate its 
strength, through the calculation of excited state lifetimes in atomic species using time-dependent density functional theory (TDDFT) in real time. The accuracy obtained for this property, whose realistic estimation is a theoretical challenge, demonstrates the predictive power of the formalism.

Formulation. - For a set of electrons with meanfield interactions, described by the wave-functions $\left\{\left|\phi_{j}\right\rangle\right\}$, with a Hamiltonian $\hat{H}=-\hbar^{2} \nabla^{2} / 2 m+\hat{V}_{e}\left(\hat{V}_{e}\right.$ is the potential energy operator) and fixed nuclei, the Lagrangian assumes the form

$$
L=i \hbar \sum_{j}\left\langle\phi_{j} \mid \dot{\phi}_{j}\right\rangle-E_{e}
$$

where $E_{e}$ is the electronic energy. In the presence of forces not arising from a potential, as in the case of friction in viscous media, the Lagrange equations with generalized coordinates $|\phi\rangle$ and $|\dot{\phi}\rangle$ read

$$
\frac{d}{d t}\left(\frac{\partial L}{\partial\left|\dot{\phi}_{j}\right\rangle}\right)-\frac{\partial L}{\partial\left|\phi_{n}\right\rangle}+\frac{\partial F}{\partial\left|\dot{\phi}_{n}\right\rangle}=0
$$

where $F$ is Rayleigh's dissipation function $[42,43]$. In classical mechanics it expresses the rate of energy dissipation due to friction. To introduce the energy lost by the electrons in the form of electromagnetic radiation, $F$ can be set equal to one half the radiation power $P_{\text {rad }}(t)$, which in turn can be written for an arbitrary charge distribution in terms of the time derivative of the dipole moment $\mu[44]$ :

$$
P_{\text {rad }} \cong \frac{\mu_{0}}{6 \pi c}[\ddot{\mu}(t)]^{2}=2 F,
$$

where $c$ and $\mu_{0}$ are the speed of light and the magnetic permeability. Then the dissipative contribution is:

$$
\frac{\partial F}{\partial\left|\dot{\phi}_{n}\right\rangle}=\frac{1}{2} \frac{\partial P_{r a d}}{\partial\left|\dot{\phi}_{n}\right\rangle}=\frac{\mu_{0}}{6 \pi c} \ddot{\mu} \cdot \frac{\partial \ddot{\mu}}{\partial\left|\dot{\phi}_{n}\right\rangle} .
$$

The dipole for the electronic system and its derivative are expressed as

$$
\begin{gathered}
\mu(t)=\sum_{j}\left\langle\phi_{j}|\hat{\mu}| \phi_{j}\right\rangle, \\
\frac{\partial \mu}{\partial t}=\sum_{j}\left\langle\dot{\phi}_{j}|\hat{\mu}| \phi_{j}\right\rangle+\sum_{j}\left\langle\phi_{j}|\hat{\mu}| \dot{\phi}_{j}\right\rangle
\end{gathered}
$$

where $\hat{\mu}=e \hat{x}$ is the dipole operator, with $e$ the electron charge and $\hat{x}$ the position operator (extension to $3 \mathrm{D}$ is discussed in the Supplemental Material [45]). To develop this expression further, we rewrite the time-derivative of the wave-functions in terms of a Schrödinger equation of the form

$$
|\dot{\phi}\rangle=-\frac{i}{\hbar} \tilde{H}|\phi\rangle
$$

with $\tilde{H}$ a time-dependent Hamiltonian that incorporates radiative emission - and which remains unknown for now. This leads to

$$
\frac{\partial \mu}{\partial t}=\sum_{j} \frac{i}{\hbar}\left\langle\phi_{j}|[\tilde{H}, \hat{\mu}]| \phi_{j}\right\rangle
$$

$$
\frac{\partial^{2} \mu}{\partial t^{2}}=\frac{i}{\hbar} \sum_{j}\left\langle\dot{\phi}_{j}|[\tilde{H}, \hat{\mu}]| \phi_{j}\right\rangle+\left\langle\phi_{j}|[\tilde{H}, \hat{\mu}]| \dot{\phi}_{j}\right\rangle+\left\langle\phi_{j}|[\dot{\tilde{H}}, \hat{\mu}]| \phi_{j}\right\rangle,
$$

and therefore the dissipative contribution becomes

$$
\frac{\partial F}{\partial\left|\dot{\phi}_{n}\right\rangle}=\frac{i}{\hbar} \frac{\mu_{0}}{6 \pi c} \ddot{\mu} \cdot \frac{\partial}{\partial\left|\dot{\phi}_{n}\right\rangle}\left[\sum_{j}\left\langle\phi_{j}|[\tilde{H}, \hat{\mu}]| \dot{\phi}_{j}\right\rangle\right] .
$$

Collecting this derivative together with the other terms in equation 2, taking the Hermitian conjugate, and equating to zero, for the evolution of the wave-functions we obtain

$$
\left|\dot{\phi}_{n}\right\rangle=-\frac{i}{\hbar} \hat{H}\left|\phi_{n}\right\rangle-\frac{\mu_{0}}{6 \pi c \hbar^{2}} \ddot{\mu} \cdot[\hat{\mu}, \tilde{H}]\left|\phi_{n}\right\rangle .
$$

Now, for equations 7 and 11 to be consistent, the following relation should hold:

$$
\tilde{H}=\hat{H}+\frac{\mu_{0}}{i 6 \pi c \hbar} \ddot{\mu} \cdot[\hat{\mu}, \tilde{H}],
$$

or

$$
\tilde{H}=\hat{H}+\frac{\mu_{0}}{i 6 \pi c \hbar} \ddot{\mu} \cdot[\hat{\mu}, \hat{H}]+\left(\frac{\mu_{0}}{i 6 \pi c \hbar} \ddot{\mu}\right)^{2} \cdot[\hat{\mu},[\hat{\mu}, \hat{H}]]+\cdots
$$

Terms in this series scale as powers of the fine-structure constant $\alpha=\frac{\mu_{0} e^{2} c}{4 \pi \hbar}$, where $c$ is the speed of light. Considering $\alpha$ as a small parameter, we truncate it after the second term [46], whereupon the effective Liouville equation for the electronic density matrix becomes

$$
\hbar \frac{\partial \hat{\rho}}{\partial t}=-i[\hat{H}, \hat{\rho}]-\frac{A}{\hbar}[[\hat{\mu}, \hat{H}], \hat{\rho}]
$$

with $A=\frac{\mu_{0}}{6 \pi c} \ddot{\mu}$. This is our fundamental equation of motion.

Validation. - To assess the properties of equation 14, simulations of the evolution of the density matrix in time were performed, employing a nearest neighbour tightbinding (TB) model. Details of the Hamiltonian and the time-integration algorithm are provided in the Supplemental Material. This model satisfies the main assumptions underlying Fermi's golden rule, which provides a framework to validate the outcome of the quantum simulations. Results for a two level system are presented in Fig. 1. The electron density is allowed to evolve starting from an excited state and suppressing the dissipation term during the first steps. The dissipation is turned on at $\sim 20$ fs, causing a decrease of the total - or electronicenergy that, after an initial lag, exponentially decays to 


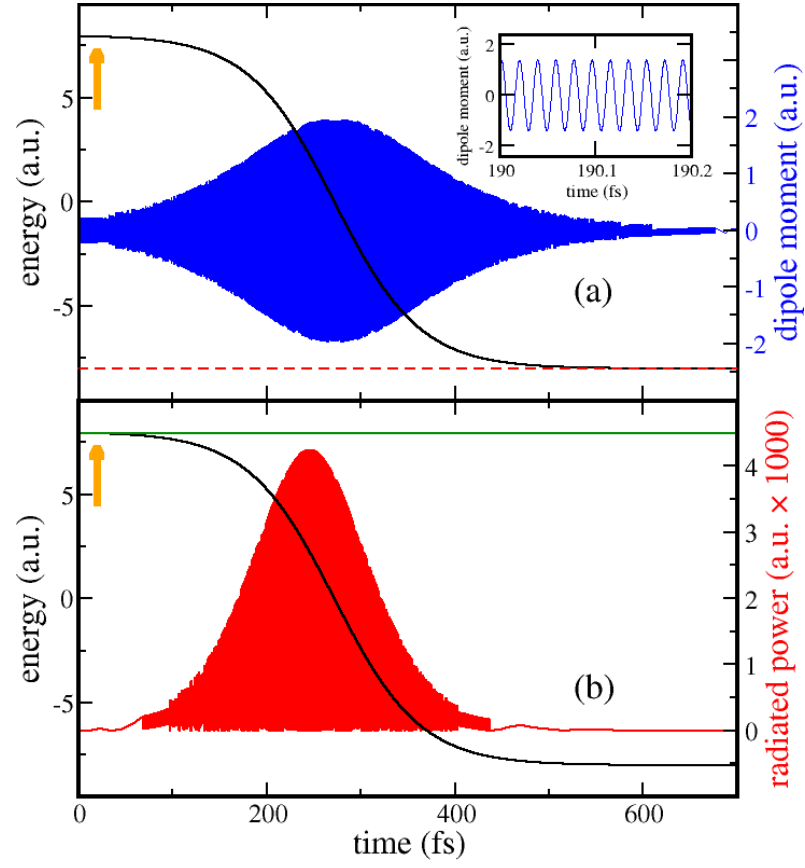

FIG. 1: (a) Electronic energy (black) and dipole moment (blue) as a function of time for a two atom molecule simulated with a TB model and equation 14. The dynamics was started from an excited state in the absence of dissipation (see text), which is turned on at $\sim 20 \mathrm{fs}$, at the point indicated by the orange arrow. The red dashed line marks the ground state level. (b) The electronic energy (black) is shown for the same system along with the radiated power (red) calculated from the Larmor formula. The green line depicts the temporal integral of the radiated power plus the electronic energy.

the ground state level. Panel (a) shows the overshooting of the dipole moment oscillations coinciding with the deexcitation: this is the classical fingerprint of photon emission. As the system relaxes to the ground state the dipole fluctuations are quenched and eventually vanish.

Under equation 14, a stationary solution will remain at rest, since a constant dipole implies $\ddot{\mu}=0$, which suppresses the dissipative term, consistent with the physics behind the model. In the present example the departing state is an excited configuration (exc) where the highest energy orbital is replaced by a coherent mixture of the HOMO and LUMO of the ground state (gs),

$$
\left|\phi_{n}^{e x c}\right\rangle=\cos \left(\frac{\theta \pi}{2}\right)\left|\phi_{n}^{g s}\right\rangle+\sin \left(\frac{\theta \pi}{2}\right)\left|\phi_{n+1}^{g s}\right\rangle
$$

with $n$ equal to the number of filled orbitals. In the spinless system explored in Fig. 1, where $n=1$ and $\theta=0.96$, the initial state is very close to the first excited eigenstate. The low amplitude fluctuations of the dipole moment visible at the start in Fig. 1a result from the propagation of this non-stationary state.

Panel (b) presents the radiated power in red, together with the sum of the electronic and dissipated energies in green, where the latter was computed as the time-integral of the emitted power. In the dissipative dynamics this sum is a conserved quantity, evincing the consistency of the model. This follows from the energy balance

$$
\frac{d\langle\hat{H}\rangle}{d t}=\frac{1}{i \hbar}\langle[\hat{H}, \tilde{H}]\rangle=-\frac{A}{\hbar^{2}}\langle[\hat{H},[\hat{\mu}, \tilde{H}]]\rangle \approx-\frac{\mu_{0}}{6 \pi c} \ddot{\mu}^{2},
$$

where we have expanded $\ddot{\mu}$ to first order in $A$. This outcome for $d\langle\hat{H}\rangle / d t$ is the radiation rate according to the Larmor formula given in equation 3.

For a two level Hamiltonian with eigenstates $|a\rangle$ and $|b\rangle$, and eigenvalues $E_{a}$ and $E_{b}=E_{a}+\hbar \omega_{b a}$, it is possible to show that the relaxation is determined by a rate $\Gamma=\frac{4 \alpha}{3 c^{2}} \omega_{b a}^{3}|\langle a|\hat{x}| b\rangle|^{2}$ as predicted by FGR for spontaneous emission (see Supplemental Material). Simulations confirm that also in many-electron molecules the occupations evolve in quantitative agreement with spontaneous emission. Comparison with the occupations deduced from FGR for systems of different sizes and interactions show that the tails of the simulated curves closely reproduce the theoretical decay rates, regardless of the departing state; e.g. $\Gamma_{\text {sim }}=7.39 \times 10^{-4} \mathrm{fs}^{-1}$ vs $\Gamma_{F G R}=$ $7.42 \times 10^{-4} \mathrm{fs}^{-1}$ for a ten atom molecule (see Supplemental Material).

In Fig. 2 we present results for the deexcitation of a four atom, one electron system, initially at the highest energy state generated from a linear combination of the third and fourth eigenfunctions, $\left|\phi_{n}^{e x c}\right\rangle=\cos \left(\frac{\theta \pi}{2}\right)\left|\phi_{j}^{g s}\right\rangle+$ $\sin \left(\frac{\theta \pi}{2}\right)\left|\phi_{k}^{g s}\right\rangle$ with $n=k=4, j=3$, and $\theta=0.96$. We see a cascade where excited states are sequentially populated, one at a time, consistent with the symmetry allowed transitions. The system approaches an eigenstate hovering over it before relaxing to the next lower level, eventually reaching the ground state. Notwithstanding how close the wave-function comes to these stationary solutions, which suppress the commutator of $\hat{H}$ and $\hat{\rho}$, the dipole fluctuations drive the dynamics forward. The dipole oscillations are present throughout the process, increasing during the transit from one eigenstate to the next, fading completely after landing in the ground state.

Fig. 2b depicts the power spectrum computed from the Fourier transform of the dipole moment. The two sharp peaks reflect the three allowed transitions, of which $4 \rightarrow 3$ and $2 \rightarrow 1$ are degenerate. Two outcomes stand out: (i) The ratio between the heights of the two peaks, equal to 3.38 , compares well with the ratio based on spontaneous emission, of 3.56. In the Supplemental Material it is shown that similar agreement is found for oscillator strengths in the absorption spectra produced through monochromatic illumination or excitation with a pulse of white light. (ii) The computed lineshapes reproduce the Lorentzians characteristic of natural broadening. The blue curves in the insets of Fig. $2 b$ represent the expected profiles according to spontaneous emission.

Excited states lifetimes in atomic species. - The ac- 


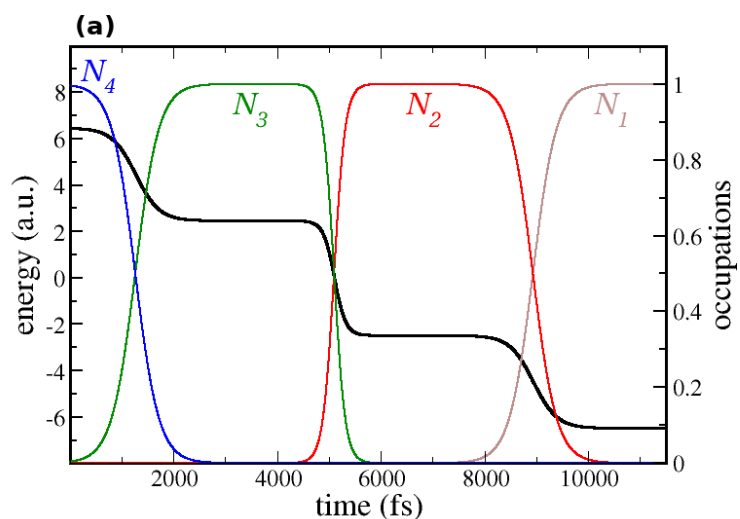

(b)

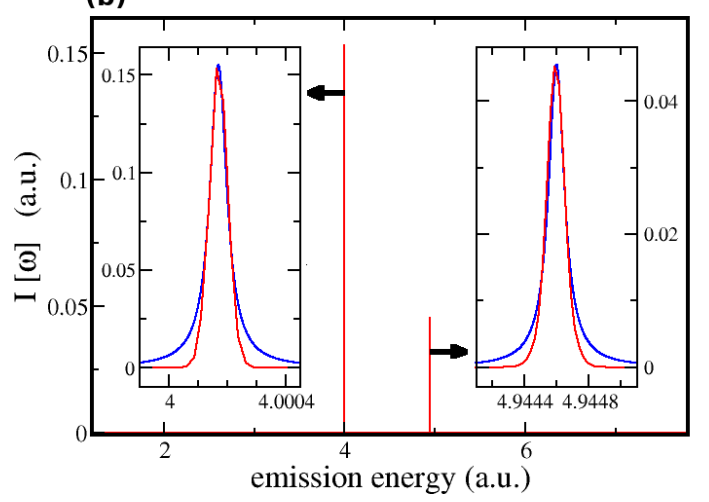

FIG. 2: (a) Electronic energy (black) and populations $\left(N_{1}\right.$, $N_{2}, N_{3}$ and $N_{4}$ ) as a function of time for a single-electron, four-atoms TB model, departing from the highest energy excited state (see text). The eigenvalues are $-6.4721,-2.4721$, 2.4721, and 6.4721 a.u. (b) Power spectrum of the relaxation process encompassing the $3 \rightarrow 2$ and $2 \rightarrow 1$ transitions. In the insets, the spectral lineshapes (red) fit the Lorentzian profiles (blue) inherent to natural broadening.

curate determination of radiative decay rates of electronic excitations in atoms and ions, of primary relevance in atomic physics and spectroscopy and fundamental assets in astrophysics, represents a major challenge for both experiment and theory. The theoretical side involves highly-demanding schemes such as multi-configuration Dirac-Hartree-Fock theory or multiconfiguration Hartree-Fock theory, in the latter case followed by the Breit-Pauli treatment [47]. Hence, the assessment of fluorescence lifetimes $(\tau)$ of atomic species entails a stringent test of our development. To this end, equation 14 was implemented in a real time TDDFT code developed in our group based on Gaussian functions and pseudopotentials [48-50]. Details are given in the Supplemental Material. With this approach the dissipative quantum dynamics of a many-electron system can be evolved from first-principles. An obstacle to simulate fluorescence at this level of theory is that the associated lifetimes typically fall in the order of nanoseconds, much above the feasibility of TDDFT dynamics. To overcome this difficulty, simulations were performed with an accel- eration factor $f$ in the equation of motion:

$$
\hbar \frac{\partial \hat{\rho}}{\partial t}=-i[\hat{H}, \hat{\rho}]-f \frac{A}{\hbar}[[\hat{\mu}, \hat{H}], \hat{\rho}] .
$$

For each factor $f$ a different decay rate $\Gamma(f)$ is extracted. Then, the rate corresponding to the non-accelerated evolution, $f=1$, is extrapolated from a plot of $\Gamma(f)$ versus $f$. Fig. 3 illustrates this treatment for the $2 s 2 p\left({ }^{1} P\right) \rightarrow 2 s^{2}$ transition in the beryllium atom. The curves in Fig. 3a that result from the excitation with a short electric pulse exhibit an exponential decay of the occupancies for $f$ in the range $5 \times 10^{4}-5 \times 10^{5}$ (details in the Supplemental Material). It can be seen in Fig. $3 \mathrm{~b}$ that $\tau^{-1}$ varies linearly with $f$, which facilitates a reliable extrapolation. This linear relationship between the decay rate and the acceleration factor can be formally derived for a two-level system (see Supplemental Material), suggesting that it is not an accident linked to this particular case, but arises from the structure of the coupling term and holds in general. The resulting lifetime of $1.97 \mathrm{~ns}$ is consistent with the experimental values, reported in the range $1.77-2.5$ ns [51].

The same procedure was applied to the Be isoelectronic species $\mathrm{B}^{+}$and $\mathrm{C}^{2+}$. This isoelectronic series has been thoroughly characterized in the literature, both from theory and experiments, and at the same time its excitations are well described by TDDFT. These circumstances make these species an appropriate set for benchmarking. The extrapolated lifetimes for $\mathrm{B}^{+}$and $\mathrm{C}^{2+}$ (Fig. 3b) are 0.831 and 0.558 ns respectively, in impressive agreement with the available data from beam-foil spectroscopy, of $0.86 \pm 0.07$ and $0.57 \pm 0.02 \mathrm{~ns}[52,53]$.

Excitation energies from linear-response TDDFT for these systems exhibit errors in the order of $5 \%$ (see Supplemental Material). In light of this, the accord between simulations and experiments - matching or excelling the one obtained through much more costly multiconfigurational quantum chemistry methods - may seem surprising and even puzzling. We conjecture that the reason behind this accuracy is that the decay process is determined by the acceleration of the dipole, and not by the dipole itself. Whereas TDDFT may fail to reproduce the exact magnitude of the dipole moment - in the same way that it may not get the right position of a spectral signal but can reasonably predict its shift upon a change in the environment [3] - it is seemingly good enough to describe the dipole variations, which dominate the relaxation dynamics. However, the dynamical description will be subject to the electronic structure method: the quality of the simulations will be conditioned by the ability of the Hamiltonian to reproduce the electronic excitations.

Final remarks. - We develop a formalism for coherent radiative electron dynamics which quantitatively captures the physics of spontaneous emission with no parameters. Even so, in this framework emission is not truly spontaneous since pure eigenstates remain station- 

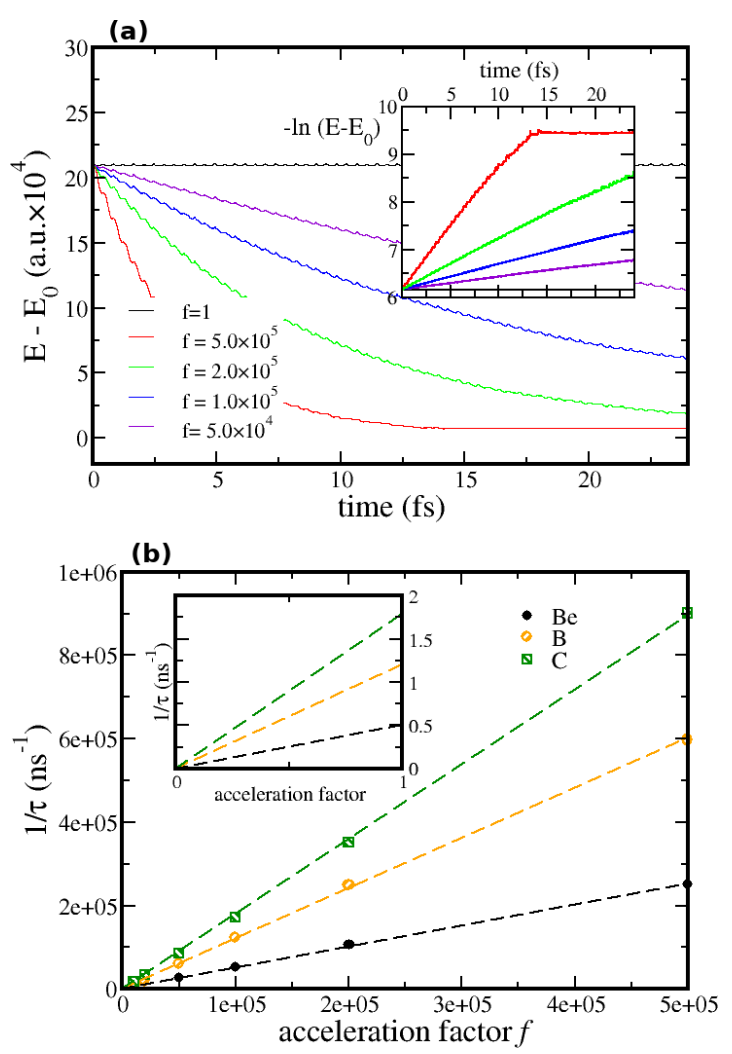

FIG. 3: Results from first-principles TDDFT simulations for the deexcitation of the $2 s 2 p$ state in $\mathrm{Be}, \mathrm{B}^{+}$and $\mathrm{C}^{2+}$, in response to an applied field. (a) Electronic energy (relative to the ground state) as a function of time for Be with different accelerations factors $f$, see equation 17 . The corresponding logarithmic plots are portrayed in the inset. (b) Inverse lifetime as a function of the acceleration factor $f$ for $\mathrm{Be}, \mathrm{B}^{+}$ and $\mathrm{C}^{2+}$. The dashed lines correspond to the linear fits. The inset shows the $y$-axis intercept for $f=1$, which inverse corresponds to $\tau$.

ary, and some perturbation is needed to trigger the process. This is inherent to the underlaying semiclassical model grounded in the Larmor equation. Alternatively, in defining the Rayleigh dissipation function, each particle could be envisioned as an independent emitter. Then equation 3 would become $\frac{\mu_{0}}{6 \pi c} \sum_{i}\left[\ddot{\mu}_{i}(t)\right]^{2}=$ $2 F$. This might be interesting for certain open-shell systems where, as observed in preliminary tests, the dipoles of two electrons can cancel, quenching the dynamics. It could also be useful in the case of two separate charge distributions - for example, two excited molecules - whose radiation will be represented as originating from a single average dipole. Such a scheme, plus the inclusion of quantum-mechanical spontaneous emission - for example via stochastic fluctuations - will be the subject of future investigations.

This is a powerful and efficient formulation that can be adapted to any Hamiltonian and which has permitted us to realize - to the best of our knowledge for the first time - realistic simulations of fluorescence. Its success underscores that the electromagnetic energy dissipation from excited electrons can indeed be described for most purposes as dipolar classical radiation. Beyond situations involving strong photon-electron coupling, the validity of our formalism is connected with that of the Larmor formula, which describes radiation from an arbitrary charge distribution. Assumptions of the Larmor formula are quite general [44], implying that our model will perform properly in situations from weak to moderate light-matter interaction, which include most applications of interest in the molecular and materials sciences (the limitations of this approach are discussed in the Supplemental Material). Thus, this formalism widens the scope of first-principles time-dependent quantum simulations, opening the door to the real-time modeling of a diversity of photophysical processes inaccessible to current approaches, from resonance energy transfer to timeresolved spectroscopy and photoluminescence.

We are grateful to Lorenzo Stella and Cristian G. Sánchez for important discussions. This work has been funded by the European Union's Horizon 2020 research and innovation programme through the project ATLANTIC under grant agreement No 823897. We acknowledge the Thomas Young Centre under grant number TYC-101, and Agencia Nacional de Promoción Científica y Tecnológica de Argentina (PICT 2015-2761 and PICT 2016-3167).

[1] M. Schultze, K. Ramasesha, C. Pemmaraju, S. Sato, D. Whitmore, A. Gandman, J. S. Prell, L. J. Borja, D. Prendergast, K. Yabana, et al., Science 346, 1348 (2014).

[2] A. Sommer, E. M. Bothschafter, S. A. Sato, C. Jakubeit, T. Latka, O. Razskazovskaya, H. Fattahi, M. Jobst, W. Schweinberger, V. Shirvanyan, et al., Nature 534, 86 (2016).

[3] U. N. Morzan, D. J. Alonso de Armiño, N. O. Foglia, F. Ramírez, M. C. González Lebrero, D. A. Scherlis, and D. A. Estrin, Chem. Rev. 118, 4071 (2018).

[4] O. A. Douglas-Gallardo, M. Berdakin, T. Frauenheim, and C. G. Sánchez, Nanoscale 11, 8604 (2019).

[5] L. Yan, F. Wang, and S. Meng, ACS Nano 10, 5452 (2016).

[6] F. Ramírez, G. Díaz Mirón, M. González Lebrero, and D. A. Scherlis, Theor. Chem. Acc. 137, 124 (2018).

[7] S. K. Min, F. Agostini, I. Tavernelli, and E. K. U. Gross, J. Phys. Chem. Lett. 8, 3048 (2017).

[8] K. Falahati, H. Tamura, I. Burghardt, and M. HuixRotllant, Nat. Commun. 9, 4502 (2018).

[9] C. G. Sánchez, M. Stamenova, S. Sanvito, D. R. Bowler, A. P. Horsfield, and T. N. Todorov, J. Chem. Phys. 124, 214708 (2006).

[10] E. J. McEniry, D. R. Bowler, D. Dundas, A. P. Horsfield, C. G. Sánchez, and T. N. Todorov, J. Phys-Condens. Mat. 19, 196201 (2007). 
[11] S. Chen, Y. Zhang, S. Koo, H. Tian, C. Yam, G. Chen, and M. A. Ratner, J. Phys. Chem. Lett. 5, 2748 (2014).

[12] U. N. Morzan, F. F. Ramírez, M. C. González Lebrero, and D. A. Scherlis, J. Chem. Phys. 146, 044110 (2017).

[13] C. M. Bustamante, F. F. Ramírez, C. G. Sánchez, and D. A. Scherlis, J. Chem. Phys. 151, 084105 (2019).

[14] D. Dundas, E. J. McEniry, and T. N. Todorov, Nat. Nanotechnol. 4, 99 (2009).

[15] O. V. Prezhdo, W. R. Duncan, and V. V. Prezhdo, Prog. Surf. Sci. 84, 30 (2009).

[16] T. Nelson, S. Fernandez-Alberti, A. E. Roitberg, and S. Tretiak, Acc. Chem. Res. 47, 1155 (2014).

[17] S. M. Falke, C. A. Rozzi, D. Brida, M. Maiuri, M. Amato, E. Sommer, A. De Sio, A. Rubio, G. Cerullo, E. Molinari, et al., Science 344, 1001 (2014).

[18] F. F. Ramirez, C. M. Bustamente, M. C. González Lebrero, and D. A. Scherlis, J. Chem. Theory Comput. 16, 2930 (2020).

[19] M. Ruggenthaler, F. Mackenroth, and D. Bauer, Phys. Rev. A 84, 042107 (2011).

[20] M. Ruggenthaler, J. Flick, C. Pellegrini, H. Appel, I. V. Tokatly, and A. Rubio, Phys. Rev. A 90, 012508 (2014).

[21] I. V. Tokatly, Phys. Rev. Lett. 110, 233001 (2013).

[22] C. Pellegrini, J. Flick, I. V. Tokatly, H. Appel, and A. Rubio, Phys. Rev. Lett. 115, 093001 (2015).

[23] M. Snchez-Barquilla, R. E. F. Silva, and J. Feist, The Journal of Chemical Physics 152, 034108 (2020).

[24] G. Khitrova, H. Gibbs, M. Kira, S. W. Koch, and A. Scherer, Nat. Phys. 2, 3026 (2006).

[25] J. Flick, M. Ruggenthaler, H. Appel, and A. Rubio, Proc Natl Acad Sci USA 114, 3026 (2017).

[26] N. Hoffmann, H. Appel, A. Rubio, and N. Maitra, Eur. Phys. J. B 91, 180 (2018).

[27] A. Abedi, E. Khosravi, and I. Tokatly, Eur. Phys. J. B 91, 194 (2018).

[28] L. Lacombe, N. Hoffmann, and N. Maitra, Phys. Rev. Lett. 123, 083201 (2019).

[29] A. Fratalocchi, C. Conti, and G. Ruocco, Phys. Rev. A 78, 013806 (2008).

[30] M. Sukharev and A. Nitzan, Phys. Rev. A 84, 043802 (2011).

[31] Y. Li, S. He, A. Russakoff, and K. Varga, Phys. Rev. E 94, 023314 (2016).

[32] D. J. Trivedi, D. Wang, T. W. Odom, and G. C. Schatz, Phys. Rev. A 96, 053825 (2017).

[33] S. Yamada, M. Noda, K. Nobusada, and K. Yabana, Phys. Rev. B 98, 245147 (2018).

[34] T. E. Li, A. Nitzan, M. Sukharev, T. Martinez, H.-T. Chen, and J. E. Subotnik, Phys. Rev. A 97, 032105 (2018).

[35] H.-T. Chen, T. E. Li, A. Nitzan, and J. E. Subotnik, Phys. Rev. A 100, 010101 (2019).

[36] T. E. Li, H.-T. Chen, and J. E. Subotnik, J. Chem. Theory Comput. 15, 1957 (2019).

[37] H.-T. Chen, T. E. Li, M. Sukharev, A. Nitzan, and J. E. Subotnik, J. Chem. Phys. 150, 044102 (2019).

[38] T. Li, H.-T. Chen, A. Nitzan, and J. Subotnik, Phys. Rev. A 101, 033831 (2020).
[39] N. M. Hoffmann, C. Schäfer, A. Rubio, A. Kelly, and H. Appel, Phys. Rev. A 99, 063819 (2019).

[40] S. A. Rashkovskiy, Indian J. Phys. 91, 607 (2017).

[41] S. A. Rashkovskiy, Indian J. Phys. 92, 289 (2018).

[42] H. Goldstein, C. Poole, and J. Safko, Classical Mechanics (Pearson, 2014).

[43] E. Minguzzi, Eur. J. Phys. 36, 035014 (2015).

[44] D. J. Griffiths, Introduction to Electrodynamics (Pearson, 2013).

[45] See Supplemental Material at http://link.aps.org/supplemental/XXXX for details about the TB and TDDFT models, propagation algorithms, theoretical background on absorption spectra, results for tight-binding occupations and oscillator strengths, data corresponding to the extrapolation of excited states lifetimes for $\mathrm{Be}, \mathrm{B}+$ and $\mathrm{C} 2+$, and a discussion on the validity of the approach in the context of an alternative derivation. Supplemental Material includes Refs. [54-60].

[46] The prefactor in the series assumes the form $\frac{2}{3} \alpha \ddot{\mu} / c^{2}$. This scales as $1 / c$, which allows us to treat $\alpha$ as a small parameter, and therefore truncate the expansion to first order. For the second order term to become significant, the acceleration should be unphysically high.

[47] C. F. Fischer, G. Tachiev, and A. Irimia, Phys. Scr. T120, 66 (2005).

[48] M. A. Nitsche, M. Ferreria, E. E. Mocskos, and M. C. G. Lebrero, J. Chem. Theory Comput. 10, 959 (2014).

[49] J. P. Marcolongo, A. Zeida, J. A. Semelak, N. O. Foglia, U. N. Morzan, D. A. Estrin, M. C. González Lebrero, and D. A. Scherlis, Front. Chem. 6, 70 (2018).

[50] U. N. Morzan, F. F. Ramírez, M. B. Oviedo, C. G. Sánchez, D. A. Scherlis, and M. C. Gonzalez Lebrero, J. Chem. Phys. 140, 164105 (2014).

[51] X. Wang, P. Quinet, Q. Li, Q. Yu, Y. Li, Q. Wang, Y. Gong, and Z. Dai, J. Quant. Spectrosc. Ra. 212, 112 (2018).

[52] S. Bashkin, L. McIntyre, H. Buttlar, J. Ekberg, and I. Martinson, Nucl. Instrum. Meth. Phys. Res. B9, 593 (1985).

[53] N. Reistad, R. Hutton, A. E. Nilsson, I. Martinson, and S. Mannervik, Phys. Scr. 34, 151 (1986).

[54] A. P. Horsfield, D. R. Bowler, A. J. Fisher, T. N. Todorov, and C. G. Sánchez, Journal of Physics: Condensed Matter 16, 8251 (2004).

[55] A. P. Horsfield, D. R. Bowler, A. J. Fisher, T. N. Todorov, and C. G. Sánchez, Journal of Physics: Condensed Matter 17, 4793 (2005).

[56] The LIO Project, https://github.com/MALBECC/LIO.

[57] J. P. Perdew, K. Burke, and M. Ernzerhof, Phys. Rev. Lett. 77, 3865 (1996).

[58] W. J. Stevens, M. Krauss, H. Basch, and P. G. Jasien, Can. J. Chem. 70, 612 (1992).

[59] G. Tachiev and C. F. Fischer, J. Phys. B: At. Mol. Opt. Phys. 32, 5805 (1999).

[60] M.-C. Kim, E. Sim, and K. Burke, Phys. Rev. Lett. 111, 073003 (2013). 